\title{
Comprehensive Mega Network(CMN) Platform: Korea MTS Governance for CIS Case Study
}

\author{
Zamira Ashurova ${ }^{1}$, Seunghwan Myeong ${ }^{1}$, \\ Alexei Tikhomirov ${ }^{1}$ \\ ${ }^{1}$ Dept. of Global e-governance \\ Inha University \\ Incheon, Republic of Korea \\ Andrey Trufanov ${ }^{2}$, Nikolay Kinash ${ }^{2}$ \\ ${ }^{2}$ Dept. of Mechanical Engineering \\ Irkutsk National Research Technical University \\ Irkutsk, Russia
}

\author{
Olga Berestneva ${ }^{3}$ \\ ${ }^{3}$ Institute of Cybernetics \\ National Research Tomsk Polytechnic University \\ Tomsk, Russia
}

Alessandra Rossodivita ${ }^{4}$

${ }^{4}$ Medical and University Center

Hospital Sacco

Milan, Italy

\begin{abstract}
Medical tourism is one of the fastest growing sectors and has a serious economic potential for Korean economy through expansion of Korean medical tourism service (MTS) network for CIS region. Existing explorations are focusing on specific areas such as economic, medical, marketing and other aspects of the medical tourism. A few academic studies devoted to governance aspects of this complex sector. The paper suggesting a comprehensive mega network is modeling framework for investigating governance process in the field of MTS delivery.
\end{abstract}

Keywords-meganetwork, CMN, MTS, stem network

\section{INTRODUCTION}

Medical tourism is one of the fastest growing sectors and has a serious economic potential for Korean economy through expansion of Korean medical tourism service (MTS) network for CIS region. Existing explorations are focusing on specific areas such as economic, medical, marketing and other aspects of the medical tourism. A few academic studies devoted to governance aspects of this complex sector. In particular, there are a lot of governance peculiarities related to transition specific to market oriented structures. In this regard a review of the current governance and medical tourism literature was made and observed an absence of a comprehensive methodological framework for analysis of the governance of medical tourism service delivery (MTSD). The subject has a networked nature and required thorough coordination of many actors and therefore the networked approach. This study aims to fill up this gap by suggesting and engineering a comprehensive mega network modeling framework for investigating the medical tourism service delivery governance process. The framework is focusing on enhancing coordination of the organizational structure mechanisms of the demand and supply sides in order to deliver medical tourism services. The study has implications for future research, and also provides valuable information for practitioners in the medical tourism sector.

\section{METHODS}

This study suggesting a comprehensive mega network is modeling framework for investigating governance process in the field of medical tourism service delivery. This approach reflecting cross sectoral and multilevel governance process of medical tourism services. Within the framework of the CMN concept it is necessary to analyze and engineer organizational mechanisms for delivery of medical tourism services and comprehensive methods and models. MTSDG is a pretty complicated system that needs a special thorough approach for its analysis. A network theory can be an efficient instrument to analyze such a multi-actor subject [1] ( Fig.1).

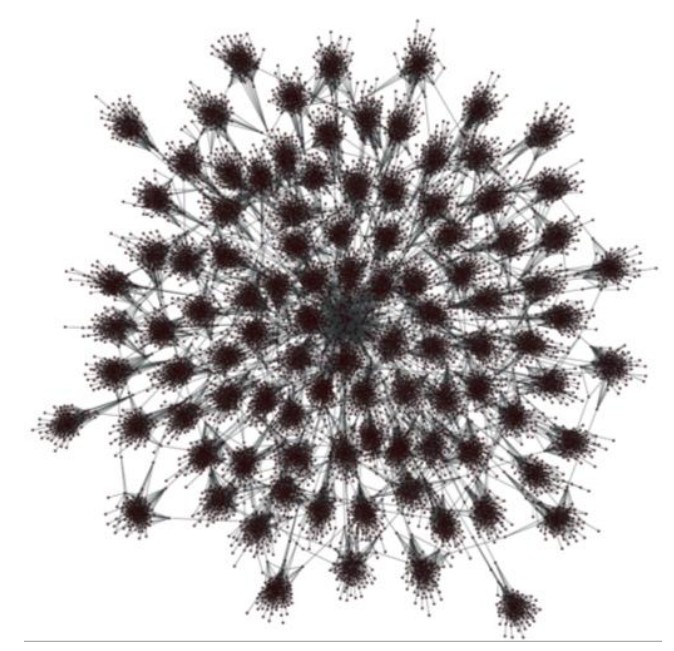

Figure 1 - Aggregate complex network as a model of large scale systems [1] 
Good network models are extremely important for studies of real complex systems. Such models should be easy enough for implementation and have limited number of metrics, while demonstrating the common properties of real systems. Moreover, models are have to be developed to check schemes, algorithms and topologies designed for massive networks. In addition, research and practical benefits are also obvious: gaining insight into the properties and processes of the real governing systems. This model approach was applied to explore the one of the biggest Social network "VK"[2]. One of the valuable fact is that in contrast to small social networks, large scale social graphs generally exhibit good expansibility. The latter property might be a platform to establish robust cooperation, promote delivery of services through national borders. Moreover, all cross disciplinary challenges intuitively utilize and enforce medical tourism network expansion. The phases of the process of governance in delivery process of medical tourism services:

1.Analysis of demand and supply in MTS

Analyses/ needs and related problems. (demand from CIS)

Analyses/ needs and related problems (Supply from ROK)

Comparison of demand and supply and decision making

2.Preparation of action programs/projects

3. Implementation of program

4.Post rehabilitation assessment

The stakeholders interaction during the governance process can be reflected in the TABLE I.

\section{TABLE I.}

\begin{tabular}{|c|c|c|c|c|c|c|c|}
\hline \multirow{3}{*}{$\begin{array}{l}\text { Phases of } \\
\text { governance process } \\
\quad \text { for MTS } \\
\quad \text { delivery } \\
\text { Governance } \\
\text { Organizational } \\
\text { structures }\end{array}$} & \multicolumn{7}{|c|}{ Analyses of demand and supply } \\
\hline & \multicolumn{3}{|l|}{ I } & \multirow{2}{*}{$\begin{array}{l}\text { III } \\
7\end{array}$} & \multirow{2}{*}{$\begin{array}{l}\text { IV } \\
8\end{array}$} & \multirow{2}{*}{$\begin{array}{l}\mathrm{V} \\
9\end{array}$} & \multirow{2}{*}{$\begin{array}{l}\text { VI } \\
10\end{array}$} \\
\hline & \begin{tabular}{l|l}
1 & 2
\end{tabular} & 3 & & & & & \\
\hline $\begin{array}{l}\text { Public sector: } \\
1 . \quad \text { High official } \\
\text { bodies } \\
\text { 2. ministries and } \\
\text { agencies } \\
\text { 3. regional bodies }\end{array}$ & & & & & & & \\
\hline $\begin{array}{l}\text { Private sector } \\
\text { and } \\
\text { CSO }\end{array}$ & & & & & & & \\
\hline
\end{tabular}

III. A DETAILED PROCEDURE COMPOSING INTERACTION OF ACTORS FROM PUBLIC AND NON PUBLIC SECTORS IN KOREA AND CIS

Analysis and selection process(I,II, III) .

1 phase. Analysis and selection process(I,II, III)

I. Analysis and selection process

1. Analyses/ needs and related problems

\section{Selection of the country}

3. Selection of the hospital

4.Selection of the doctor

II. Analysis of supply

5. Analysis of Infrastructure supply: hospitals, clinics

6. Marketing strategies on MTS policies

III.7. Comparison of CIS demand and KOREA supply

2 phase. IV. Formulation of action programs/projects

8. Preparation of action programs/projects

3 phase. Implementation and follow up

9. Implementation of program

10. Post rehabilitation assessment and reporting

\section{FINDINGS}

A special ontology that clarified thesaurus, taxonomy, semantic network was designed. Based on this ontology a pertinent Comprehensive MegaNetwork (CMN) which concentrated on principal structural links of the subject has been elaborated. CMN integrates multilevel stem networks with communities and the actors. Thus, the model for overlapping communities with heterogeneous topologies in formal and informal network layers was proposed.

\section{MTS Model}

Graph $\mathrm{G}=(\mathrm{V}, \mathrm{E})$ where V-nodes, E-links, is composing all participants engaged in governance of MTS process directly or non-directly ( doctors, patients, tourist managers, etc). For network generation R-MAT [3] has been used ( Fig 2 ). The simple model is composing one doctor per 20 patients, I patient has 10 relatives etc. The meganetwork model is, composing thousands participants of doctors, patients, tourist managers, insurance agents, pilots and flight attendants, hotel staff etc Optimization of network structure /topology and its sustainability, clusterization and security issues have been investigated.

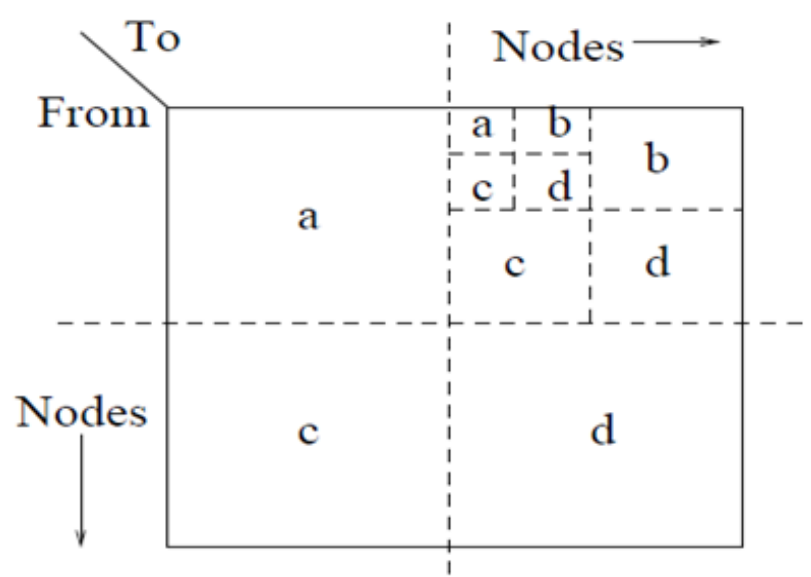

Figure 1 - R-MAT algorithm for meganetwork generation 


\section{Stem MTS Model}

Further to scientific discussion the authors within existing methodology framework developed and applied a stem network (SN) concept [4]. This concept takes into account intrinsic multilayered thematic and dynamic nature of real multi-actor systems. Multiple nodes of a multiplex networks are called stems. The stem network is described by the triple $\boldsymbol{B}=(\boldsymbol{S}, \boldsymbol{T}, \boldsymbol{C})$, "bed", where $\boldsymbol{S}$ is nonempty set of stems, $\boldsymbol{T}$ is a nonempty set of thematic layers, $\boldsymbol{C}=(\boldsymbol{C} \mathbf{1}, \boldsymbol{C 2}, \ldots, \boldsymbol{C} \boldsymbol{t})$ is a set of binary relations on the $\boldsymbol{S}$, where $\boldsymbol{C}_{\boldsymbol{i}}$ corresponds thematic layer $\boldsymbol{i}$. In traditional words SN-network is a network which is permitted to have multiple nodes, having multithread links of same nature.

The Stem MTS (CMTS )might converges humans, natural and machine agents and must coordinate and connect a huge and different varieties of them . In present paper just to model MTS a combined stem network (CSN) is proposed and defined as a specific integration of interdependent $\mathrm{SN}$ networks $\boldsymbol{V}=\left(\boldsymbol{B}_{1}, \boldsymbol{B}_{2}, . . \boldsymbol{B}_{\boldsymbol{m}}\right)$, described on $\boldsymbol{m}$ "beds" with nonoverlapping sets of stems, tematic layers and binary relations. Some stems from different "beds" might be united into a group which is called a bouquet. Such a group of real objects - multiplet (pair, triple,....) includes nonrepeatable ( as a rule) coactive stems of different beds $\left(\boldsymbol{S}_{\boldsymbol{i k}}, \boldsymbol{S}_{\boldsymbol{j}}, \ldots\right)$, where $\boldsymbol{S}_{\boldsymbol{i} \boldsymbol{k}}$ is a stem $\boldsymbol{i}$ from the bed $\boldsymbol{k}$. Thus bouquets are partitioned into stems which firm nodes of networks of the same nature. Links within a stem, between different stem nodes of the same layer of the same network, and between stems taken from different networks are put in different categories Links between the stems inside a bouquet represent binary relations (interactions) such as "dependence" ( $\boldsymbol{D}$-links), which differ from couplings - "connections" ( $\boldsymbol{C}$-links) that govern the interaction between the stems of a bed; couplings of "bindings"-type ( $\boldsymbol{B}$-links ) describe the relationship between the nodes of the same stem.

The concept categorizes system actors as stems, the latter are stratified into nodes according to each thematic layer.

Naturally Human Beings (HB) networks incorporate professional, family, and friendly connections. While transportation a container might change airlines, railways, boat lines, bus lines, etc.. It is impossible to combine human beings with gadgets by relative links neither gadgets with HB through electric signals. Such a detailing of links promotes clarification of network robustness while waiting for attacks on separate elements of a network (nodes, stems, bouquets, beds, layers, links-bindings, links-connections, and links-dependencies ) and their combinations. The novel network concept promotes easier understanding and clarification of the implicit and complicated structure the property graphs propose. Also it is of significance that all the familiar graphs: pseudographs, multigraphs, property graphs, color edged graphs bear no multiple vertices. These graph approaches concentrate on subjects of instrument traits while the combined stem network focuses on subjects of nature.

\section{Vulnerability of MTS Model}

In order to study the problems of safety and sustainable development of medical tourism service systems it has been important to take into account internal properties of stems capacities. First, capacities are needed to hold loads in a network in terms of centralities (degree centrality load, bridge centrality load, vicinity centrality load...). Second, those provide robustness while countering diverse attacks. Time factor $\mathrm{t}$ is also included into consideration for reflecting CSN dynamics. Attack schemes of the CSN structures in context of safety problems has been reviewed in frame of a simple analysis of major offensive actions, which are important for studying of SMTS structures described by combined stem networks.

The proposed model allows the following. Elements of SMTS network are considered as stems ( Fig.3) that standalone or interconnected and interdependent within and across countries, states, regions, local territories, and sites.

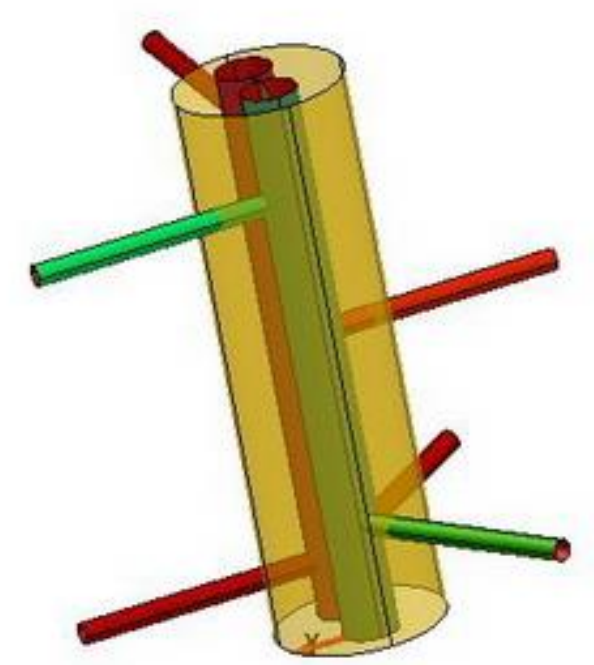

Figure 3 - 3 actors of a 2-bed Stem MTS Model

Most facilities of a technological infrastructures are proprietary of the private sector or federal, regional, or local governments, and might be stratified into diverse layers on pertinent beds while connected to other systems of the same or different field. Contrary to traditional complex networks failures and attacks a combined stem network might comprise not only removals of nodes but stems and bouquets in whole. It is evident that removal of a $\boldsymbol{C}$-link in a stem network does not necessarily tear a path between attached nodes because of $\boldsymbol{B}$-links. The recent research [5] assumed that elements in the dynamic network can fail independently of other elements (internal failure) or due to external causes ( if it has a substantially damaged neighborhood ). The study also emphasized a value of spontaneous recovery for the dynamic networks. The CSN approach is able to detail the neighborhood for further analysis of damage and spontaneous 
recovery processes concomitant to network element fails. Our model by Figure 1 also demonstrates that in case of emergency for a technological node or stem of a bouquet its "conscious" partner stem ( human or sensor ) is able to give a signal for the system to start an intentional recovery process. All these clarify CSN robustness issues and promote stem network safety strategies.

\section{CONCLUSION}

Methodological framework and comprehensive mega network concept enhancing crossectoral governance process and security issues in the medical tourism sphere was developed:

1. A comprehensive analysis of complexity for delivery of governance processes of Korean medical tourist services in CIS region was undertaken.

2. A review of existing methods and models of governance and organization in the area of medical tourism was done.

3. A methodological mechanism composing and modeling interactions of different organizations and social networks engaged in different phases of governance processes in the areas of medical tourism services was designed.

\section{REFERENCES}

[1] A.Tikhomirov, A. Afanasyev, N. Kinash, A. Trufanov, O. Berestneva, A. Rossodivita,S. Gnatyuk, R. Umerov. "Network society: aggregate topological models “, Proc. Information Technologies and Mathematical Modelling. 13th International Scientific Conference, ITMM 2014, named after A.F. Terpugov, Anzhero-Sudzhensk, Russia, November 2022, 2014. Communications in Computer and Information Science.Verlag: Springer International Publishing. vol. 487, 2014, pp.415-421

[2] N.Kinash, A. Tikhomirov, A. Trufanov, O. Berestneva, A. Boukhanovsky, Z. Ashurova. "Analysis of Large-Scale Networks Using High Performance Technology (Vkontakte Case Study)", Creativity in Intelligent, Technologies and Data Science, Volume 535 of the series Communications in Computer and Information Science,2015, pp. 531541

[3] D. Chakrabarti, Y. Zhan, and C. Faloutsos, "R-MAT: A recursive model for graph mining," in SDM '04, 2004, pp. 442-446. [Online]. URL: http://www.cs.cmu.edu/ christos/publications/siam04.pdf

[4] A.Tikhomirov, A. Trufanov. "Interconnected stem networks: Security focus". 2015, [Online]. URL: http://www.pitt.edu/ super1/lecture/lec53061/index.htm

[5] A. Majdandzic, B. Podobnik, S.V. Buldyrev, D.Y. Kenett, S. Havlin, H.E. Stanley. Spontaneous recovery in dynamical networks. Nature Physics . 10(1), 2014, pp. 34-38 